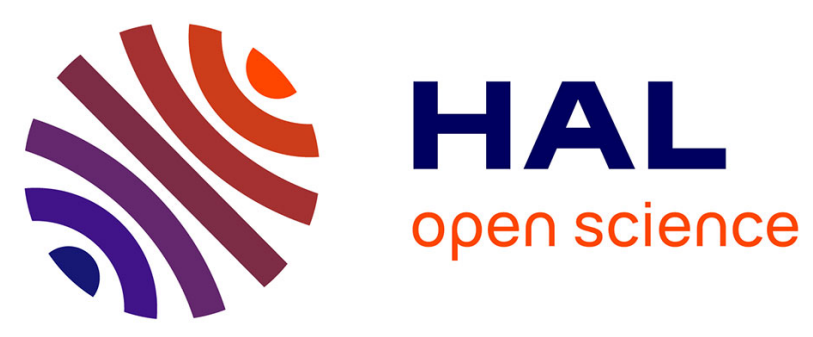

\title{
Scaling-up of Mesoporous Silica Films via an Eco-efficient UV Processing Method. Part 1: Photoinduced Mesostructuration
}

H.De Paz-Simon, C Croutxé-Barghorn, Mathilde Sibeaud, H de Paz-Simon, S. Rigolet, L. Michelin, B. Lebeau, L. Vidal, P.-A. Albouy, A. Chemtob

\section{- To cite this version:}

H.De Paz-Simon, C Croutxé-Barghorn, Mathilde Sibeaud, H de Paz-Simon, S. Rigolet, et al.. Scaling-up of Mesoporous Silica Films via an Eco-efficient UV Processing Method. Part 1: Photoinduced Mesostructuration. Microporous and Mesoporous Materials, 2018, 257, pp.42-50. 10.1016/j.micromeso.2017.08.017 . hal-02434798

\section{HAL Id: hal-02434798 https://hal.science/hal-02434798}

Submitted on 16 Jan 2020

HAL is a multi-disciplinary open access archive for the deposit and dissemination of scientific research documents, whether they are published or not. The documents may come from teaching and research institutions in France or abroad, or from public or private research centers.
L'archive ouverte pluridisciplinaire HAL, est destinée au dépôt et à la diffusion de documents scientifiques de niveau recherche, publiés ou non, émanant des établissements d'enseignement et de recherche français ou étrangers, des laboratoires publics ou privés. 


\section{Scaling-up of Mesoporous Silica Films via an Eco-efficient UV}

\section{Processing Method.}

\section{Part 1: Photoinduced Mesostructuration}

Mathilde Sibeaud $^{a, b}$, H. De Paz-Simon ${ }^{a}$, C. Croutxé-Barghorn ${ }^{a}$, S. Rigolet ${ }^{b}$, L. Michelin $^{b}$, B. Lebeau ${ }^{b}$, L. $^{2}$ Vidal $^{\text {b }, ~ P .-A . ~ A l b o u y ~}{ }^{c}$, A. Chemtob ${ }^{* b}$

${ }^{a}$ Laboratory of Macromolecular Photochemistry and Engineering, University of Haute-Alsace, ENSCMu, 3 bis rue Alfred Werner, 68093 Mulhouse Cedex, France

${ }^{\mathrm{b}}$ Institut de Sciences des Matériaux de Mulhouse, UMR-CNRS 7361, University of Haute-Alsace, 3 bis rue Alfred Werner 68093, Mulhouse Cedex, France

c Laboratoire de Physique des Solides, CNRS, UMR-8502, Université Paris-Sud, F-91405 Orsay, France

* To whom correspondence should be addressed: abraham.chemtob@uha.fr; tel: +33 389608834 


\begin{abstract}
Designing sustainable and industrially viable processing methods to synthesize ordered mesoporous films is a necessary condition to tap their full potential of applications. In order to respond to this challenge, well-established photoacid-catalyzed sol-gel photopolymerization has been harnessed to prepare large $\left(>100 \mathrm{~cm}^{2}\right)$ and micrometer-thick porous silica films possessing a 2D hexagonal mesostructure. Our UV irradiation system consists of two inexpensive and low radiant power fluorescent UV tubes $\left(3 \mathrm{~mW} \mathrm{~cm}{ }^{-2}, 280-380 \mathrm{~nm}\right.$ ) enclosed in a hygrometric chamber. Precise conditions to promote copolymer/silica hybrid film mesostructuration have been determined as regards relative humidity, film thickness and templating agent concentration. The mesostructured films have been analyzed using an extensive range of techniques including electron microscopy, grazing-incidence small-angle X-ray scattering (GISAXS), and $\mathrm{N}_{2}$ sorption measurements, and solidstate NMR spectroscopy. Mesoporous silica films with a specific surface area up to $314 \mathrm{~m}^{2} \mathrm{~g}^{-1}$ have been achieved with a very low level of microporosity. Coupling of X-ray diffraction (XRD) and FTIR spectroscopy has enabled to shed light into the photoinduced self-assembly mechanism.
\end{abstract}

Keywords: silica, radiation, ultraviolet, film, sol-gel

\title{
1. Introduction
}

25 years of intense and massive research in ordered mesoporous materials have led to two major outcomes $[1,2]$. Firstly, a thorough understanding of the self-assembly mechanism has been achieved, leading to robust processing methods to control chemical composition (silica, oxide, hybrid...), texture (pore organization, specific surface area...) and morphology (film, monolith, fiber, powder...). Secondly, their potential for delivering innovative and useful applications in extremely varied fields has been established [3-7]. Today, we are at the eve of a gradual integration of these mesoporous materials into marketable products. However, their development at very large scale will 
only be possible in the future if emerging methods readily transferable from the laboratory to the industry are introduced $[8,9]$. Although mesoporous materials are backed up with soft-chemistry solgel routes [10], scalability but also eco-design are currently two issues that have not been sufficiently raised or discussed so far. This is mainly attributed to complex experimental conditions and the high cost products involved in the synthesis [11].

The preparation of mesostructured silica or organosilica materials in the form of film is particularly representative, since it involves many constraints: multiple steps including heating for silica network stiffening and template removal, high concentration of solvents whose recovery has been rarely addressed, limited film thickness up to a few hundred nanometers only, and mostly high cost and fossil-based alkoxysilane reactants $[12,13]$. Up to now, the most complicated step is certainly to master film deposition due to a short pot-life formulation, and the need to control both the external environmental conditions - temperature, relative humidity $(\mathrm{RH})$ - as well as the deposition parameters. Nonetheless, obtaining mesoporous materials with a net-shaped film morphology is highly desired for many advanced functional applications, including sensors, supercapacitors, or photonic devices [14]. Today, a scalable, efficient, energy-saving and environmentally benign process to mesoporous silica films has yet to be developed.

In the search for environmental improvements in the processing method that could also yield parallel economic benefits, we present an eco-efficient UV process to design large surface area of micrometer-thick ordered silica mesoporous films. The concept of thin-layer radiation curing has been extensively investigated, but essentially for the preparation of organic polymer films intended for use as industrial coatings, inks and adhesives [15]. Harnessing radiation energy to synthesize inorganically cross-linked silica or metal oxide films needs to be further developed because it may provide similar technical advantages: fast production rates, low or no volatile organic compounds (VOC) formulation, and ambient temperature reaction. In 2012, UV radiation was proved to drive the synthesis of disordered silica network mesostructures [16]. More recently, ordering was even achieved upon optimizing irradiation conditions [17]. Other recent studies showed the interest of 
photoinduced inorganic polymerization for the synthesis of silica particles $[18,19]$, membranes [20], hybrid films [21, 22], and dental materials [23]. In this report, we have taken a further step forward and designed an energy-saving, scalable, and fully-photoinduced process where UV radiation has two consecutive roles: $\mathrm{i}$. the formation of silica/surfactant mesostructured film and, ii. the selective and fast photodegradation of the copolymer templating agent. For the sake of clarity, each UV process induced by two different wavelength ranges - is dealt in a separate study. This study entitled Part 1 Photoinduced Mesostructuration focuses on the first $\mathrm{UV}_{\mathrm{B}}$-driven $(280-320 \mathrm{~nm})$ condensation of methoxysilane oligomer precursors to form ordered copolymer/silica hybrid films. In this report, mesoporous silica films have, therefore, been produced by thermal calcination. A separate forthcoming study Part 2: Photoinduced Calcination will describe the second stage based on $U_{C^{-}}$ driven (160 - $280 \mathrm{~nm}$ ) calcination to synthesize the final mesoporous silica film.

Our study begins with a description of the new UV radiation system to produce silica/surfactant ordered films. The added values compared to a conventional set-up based on evaporation-induced self-assembly (EISA) methodology have been emphasized. Secondly, the photogenerated sol-gel films prepared with a triblock copolymer based on poly(ethylene oxide) (PEO) and poly(propylene oxide) (PPO) ((EO) $\left.{ }_{19}-(\mathrm{PO})_{69}-(\mathrm{EO})_{19}\right)$ have been thoroughly characterized using a range of techniques: transmission electron microscopy, X-ray diffraction (XRD), grazing-incidence small-angle X-ray scattering (GISAXS), $\mathrm{N}_{2}$ adsorption and ${ }^{29} \mathrm{Si}$ and ${ }^{1} \mathrm{H}$ solid-state NMR. The influence of copolymer concentration, relative humidity $(\mathrm{RH})$ and film thickness have been examined in a view of optimizing the process. To understand the light controlled self-assembly mechanism, XRD and FTIR have been conducted in parallel to show the interplay between the progress of the sol-gel process and the onset/evolution of the mesophases.

\section{Materials and Methods}

\subsection{Materials}


Pluronic 123 (P123), an amphiphilic triblock copolymer based on PEO and PPO ((PEO) ${ }_{19}-(\mathrm{PPO})_{69^{-}}$ $\left.(\mathrm{PEO})_{19}\right)$ and diphenyliodonium hexafluorophosphate $\left(\left.\Phi_{2}\right|^{+} \mathrm{PF}_{6}^{-}\right)$, a photoacid generator, were purchased from Sigma Aldrich. Poly(dimethoxysiloxane) (PDMOS) is an oligomeric methoxy precursor provided by $A B C R$ (Figure $\mathbf{1}$ depicts a simplified structure, and Figure $\mathbf{S} \mathbf{1}$ in supplementary material provides the ${ }^{29} \mathrm{Si}$ liquid state NMR spectrum). Polished silicon wafers from Siltronix and $10 \times 10 \mathrm{~cm}$ glass substrate from Brot were previously washed with acetone and used as substrates.

\subsection{UV processing method to mesoporous silica films}

The starting formulation is prepared by mixing PDMOS, $\mathrm{P} 123, \Phi_{2} \mathrm{I}^{+} \mathrm{PF}_{6}^{-}$and acetone with a $1 / x / 0.06 / 1$ wt. ratio $(0.1<x<1)$. The resulting homogenous and stable solution is either deposited on silicon wafer with a spin coater $(15 \mathrm{~s}$ at $1000 \mathrm{rpm}$ then $30 \mathrm{~s}$ at $10000 \mathrm{rpm})$ or onto a $10 \times 10 \mathrm{~cm}$ glass substrate using a $6 \mu \mathrm{m}$ bar coater, leading to $\sim 3.6 \mu \mathrm{m}$ films (see characterization section for details on film thickness measurement). The formulated system is photolatent when stored in a vial at ambient temperature, and insensitive to visible light. Irradiation was performed inside a Memmert HCP 108 hygrometric chamber, with a careful monitoring of temperature and $\mathrm{RH}: 30^{\circ} \mathrm{C}$ and $60 \%$ in standard conditions, respectively. Even when the samples were stored in the environmental chamber, no sign of early hydrolysis was observed within $1 \mathrm{~h}$ in absence of UV radiation. Irradiation was provided by a compact system UV 236 (dimensions: $470 \times 280 \times 100 \mathrm{~mm}$ ) placed inside the chamber. This radiation system was purchased from Waldmann SA, and includes two fluorescent tubes (UV6, 36 W, Philips). These lamps cover a broadband spectrum, spanning from 280 to $380 \mathrm{~nm}$ (see full spectrum in Figure S2), with a total irradiance of approximately $3 \mathrm{~mW} \mathrm{~cm}^{-2}$ at a distance of 6 $\mathrm{cm}$ from the lamp. After $30 \mathrm{~min}$ exposure, transparent and solid hybrid films were obtained. Calcination of the surfactant was then performed under air at $250{ }^{\circ} \mathrm{C}$ for $4 \mathrm{~h}$ with a preliminary $2 \mathrm{~h}$ step at $100^{\circ} \mathrm{C}$ (heating rate of $1{ }^{\circ} \mathrm{C} \mathrm{min}^{-1}$ ).

\subsection{Characterization}


Film thickness was measured by an optical profilometer (Altisurf 500). Two different X-ray diffraction (XRD) experiments were performed. The acquisition of simple $\theta-2 \theta$ diffractograms was performed with a X'Pert Pro (PANalytical) diffractometer using Cu Ka radiation ( $\lambda=0.15418 \mathrm{~nm} ; 0.5^{\circ}$ $\left.<2 \theta<10^{\circ} ; 0.017^{\circ} \mathrm{s}^{-1}\right)$. However, the information provided with this technique is restricted to scattering vectors perpendicular to the substrate. As result, 2D-GISAXS patterns were used additionally when it was necessary to remove ambiguities in the interpretation of the formed mesostructures. 2D-GISAXS analysis was performed on a homemade SAXS apparatus mounted on a rotating anode generator ( $\mathrm{Cu}$ K $\alpha$ radiation, $40 \mathrm{kV}, 20 \mathrm{~mA}$, small focus). The alignment of the sample was performed with position-sensitive detector so as to visualize the location of the reflected beam. The sample was oscillated by ca. $1^{\circ}$ in grazing incidence during acquisition; diffraction patterns were recorded on photostimulable imaging plates. XRD and GISAXS patterns were directly taken on the sample deposited on silicon wafer or glass substrate. Transmission FTIR spectra were obtained directly on films deposited on silicon wafer using an IR Equinox with a resolution of $4 \mathrm{~cm}^{-1}$ and 32 spectra accumulation. All spectra were baseline corrected and integrated under OPUS 7.0 software. For measuring XRD patterns and IR spectra at different irradiation times (see section 3.3), similar and minimum time intervals were taken between the sample removal from the UV chamber and itsanalysis: $5 \mathrm{~min}$ (FTIR) and $10 \mathrm{~min}$ (XRD). Such conditions are meant to limit the effect of dark postreactions (hydrolysis and condensation). Morphology of the as-synthesized and calcined products was observed by transmission electron microscope TEM (JEOL JEM-ARM200F). $\mathrm{N}_{2}$ adsorption and desorption isotherms were performed on a Tristar 3000 (Micrometrics). Calcined samples were degassed under vacuum at $150{ }^{\circ} \mathrm{C}$ during $12 \mathrm{~h}$. Surface area was determined by BET method, average pore diameter and distribution were evaluated from desorption branch by BJH method. ${ }^{29} \mathrm{Si}$ MAS NMR spectra of hybrid materials were recorded on a Bruker AVANCE II 300WB spectrometer $\left(B_{0}=7.1\right.$ T) operating at $59.61 \mathrm{MHz}$ for the ${ }^{29} \mathrm{Si}$, with $7 \mathrm{~mm}$ double resonance MAS probe at a speed of $4 \mathrm{kHz}$, with a $\pi / 6$ pulse duration of $2 \mu \mathrm{s}$, a contact time of $4 \mathrm{~ms}$, a recycle delay of $80 \mathrm{~s}$, and a high-power proton decoupling (DEC) during the acquisition. These recording conditions ensure the quantitative 
determination of the proportion of different $Q_{n}$ Si species. All the NMR experiments were performed at room temperature. ${ }^{29} \mathrm{Si}$ chemical shifts were referenced to tetramethylsilane (TMS). Assignment of NMR resonances was performed with Topspin 3.1 software, spectra deconvolutions using the DMFIT

2015 Software [24]. Condensation degrees were calculated using the equation: $C D=\sum \frac{\mathrm{n} \times \mathrm{A}\left(\mathrm{Q}_{n}\right)}{\mathrm{f}}$, where $A\left(Q_{n}\right)$ is the integrated area of the different $Q_{n}$ siloxane species of the ${ }^{29}$ Si NMR spectrum, and $f$ the functionality of the silicon atom (4).

\section{Results and Discussion}

\subsection{UV irradiation set-up and photosensitive sol-gel mixture}

Aiming at a smarter and simpler way of producing mesostructured silica films at industrial scale, our approach is eco-efficient in regards to reactants and photochemical set-up:

Reactants. Only three reactants are used (see structures in Figure 1): silica precursor, surfactant and photoacid generator (PAG), which form a photolatent formulation when mixed together. Of significant importance to ensure a long-term stability, the addition of water is not necessary since water concentration can be indirectly controlled by adjusting the RH inside the UV chamber where the reaction is triggered (see next section for details). However, a minimal amount of acetone is added to achieve a homogenous mixture. However, used at moderate content (PDMOS/Acetone: 1/1 wt ratio), this co-solvent does not hamper the one-step production of micrometer-thick films $(3.4 \mu \mathrm{m}$ under reference conditions), which is required for many coatings applications. Industrially produced, the pre-polymerized TMOS-based methyl silicate 51 (also known as poly(dimethoxysiloxane) or PDMOS) is used as silica source. Its oligomeric structure prevents evaporation, and less methanol byproduct is released because of its precondensed form. A non-ionic PEO- $b$-PPO- $b$-PEO polymeric surfactant (P123) is employed. It is known to be accessible, biodegradable and less-toxic compared to quaternary ammonium surfactants [25]. A commercial diaryl iodonium photoacid generator (PAG, $\Phi_{2} l^{+} \mathrm{PF}_{6}^{-}$) with a maximum absorption at $300 \mathrm{~nm}$ allows the reactive mixture to be photostable even 
in the presence of visible radiation provided by sunlight or artificial light. The stability of the PDMOS/P123/PAG formulation extends at least 3 months, opening the way for a single-component formulation $1 \mathrm{~K}$ highly sought after in industry. As a result of photolatency, a precise control of the deposition parameters is no longer necessary, and film surface can be reworked before UV irradiation with less waste of raw materials.

UV irradiation set-up. A second key aspect is the improvement of installation cost and size. As depicted in the photograph of Figure 1, we have used a low power UV fluorescent tube while radiation curing technology still heavily relies on more energy-intensive medium-pressure $\mathrm{Hg}$ arcs. It features low price, good conversion of electrical energy to UV radiation (30\%), emission in the UV range to match the PAG's absorption region, and very low irradiance $\left(3 \mathrm{~mW} \mathrm{~cm}{ }^{-2}\right)$ implying limited risk for the operator despite the short emitted wavelengths. Last but not least, the heat release is negligible allowing environmental conditions to be readily tailored, which is critical to self-assembly. Therefore, the photoacid-catalyzed sol-gel process can take place at constant temperature $(T=30$ $\left.{ }^{\circ} \mathrm{C}\right)$, while controlling also the $\mathrm{RH}(60 \%)$. In addition, large surface films can be produced in this climate chamber whose size can be readily up-scaled. The closed environment enables to recover methanol by-products (which has not been taken into account here) to reduce emission and pollution, thus eliminating hazardous materials from production processes.

\subsection{Photosynthesis and characterization of large silica/copolymer mesostructured films}

TEM and $\mathbf{N}_{\mathbf{2}}$ adsorption. Three silica/surfactant hybrid films including various surfactant weight ratios $(x=0.25,0.45$, and 0.55$)$ were prepared under $U V_{B}$ irradiation inside the hygrometric chamber, then thermally calcined. In Figure 2, the TEM images of the three calcined silica films clearly indicate the presence of 2D hexagonal mesophases parallel to the substrate. Such morphology should not be confused with lamellar morphology which cannot withstand a calcination process. In mesoporous film preparation, enhanced interactions of the block copolymer chains with the substrate are known 
to drive a preferential orientation of the mesostructure [26]. Clearly, a higher level of ordering was obtained upon increasing the P123 concentration. Average pore sizes of 34,41 and $50 \AA$ for $x=0.25$, 0.45 and 0.55 , respectively, were also measured from TEM images by using image-J software on about 30 measurements for each sample with a standard deviation of $1 \AA$. The resulting values are reported in Table 1 and were found in good agreement with those obtained by the BJH method applied on the desorption branches of the $N_{2}$ nitrogen adsorption isotherms (39, 38/43 and 43/48 $\AA$ for $x=0.25,0.45$ and 0.55 , respectively). Both methods are not accurate for determining pore size since pores are not regular and $\mathrm{BJH}$ method is known to underestimate small mesopore size $(<11$ $n m)$ [27]. Moreover, pore size distributions are broad and bimodal for $x=0.45$ and 0.55 samples. Nevertheless, they give two major tendencies: an average pore size of 35-50 $\mathrm{A}$ which increases with the surfactant content. The $\mathrm{N}_{2}$ adsorption isotherms yielded specific surface areas of $119(x=0.25)$, $191(x=0.45)$ and $314 \mathrm{~m}^{2} \mathrm{~g}^{-1}(\mathrm{x}=0.55)$. The adsorption and desorption plots depicted in Figure 3 reveal a type IV isotherm with a $\mathrm{H} 1$ type hysteresis, consistent with a mesoporous material containing cylindrical pores. A small shoulder on the desorption branch reflects slightly irregular pores with presumably a bottleneck shape. Such pore morphology is also supported by TEM images $(x=0.25$, Figure 2$)$ showing accordingly irregular channels. The pore size distribution (see insert of Figure 3) suggests that pore diameter increases with surfactant concentration. A bimodal distribution is observed for $x=0.45$ ( 38 and $43 \AA$ ) and $x=0.55$ ( 43 and $48 \AA$ ). In terms of pore accessibility and volume, it is worth noting that these two samples have an equivalent pore volume $\left(0.30-0.37 \mathrm{~cm}^{3} \mathrm{~g}^{-}\right.$ $\left.{ }^{1}\right)$, but $x=0.55$ has a higher BET surface area $\left(314 \mathrm{~m}^{2} \mathrm{~g}^{-1}\right)$ due to the presence of micropores $(0.03$ $\mathrm{cm}^{3} \mathrm{~g}^{-1}$ ). Microporosity is generally assigned to the inclusion of hydrophilic PEO chains into the siloxane network [28-30]. However, the level of microporosity remains relatively low in our photogenerated samples [31], accounting for the substantially lower specific surface area values compared to other mesoporous films obtained by EISA $\left(\approx 600-900 \mathrm{~m}^{2} \mathrm{~g}^{-1}\right)$ [32]. It has been demonstrated that, without these micropores, a typical mesoporous silica material such as hexagonal SBA15 exhibit a surface area of only $200-300 \mathrm{~m}^{2} \mathrm{~g}^{-1}$ [30]. 
XRD. The XRD patterns in Figure 4 complete and support the description of the mesostructure given by TEM and BET data. For the three as-synthesized films, they seem consistent with twodimensional 2D hexagonal mesochannels parallel to the substrate surface [33], since the most visible peaks are the two (100) and (200) reflections. At the lowest concentration of P123 ( $x=0.25)$, the XRD peaks are weaker, indicating a more limited mesophase ordering. At $x=0.45$, some characteristic peaks of the (non-oriented) 2D hexagonal are also visible [34], making the interpretation of the XRD patterns challenging. Calcination induces both a peak broadening and a shift of their positions, reflecting that the periodic structure has condensed and that the level of ordering has been reduced. Only the sample prepared with 45 wt\% P123 shows 3 peaks indexed as the (100), (200) and (210) reflections of a 2D hexagonal structure [35], linked by the relation $d_{100}=2 d_{200}=\sqrt{7} d_{210}$. The lattice parameter for this ratio is $89 \AA$ for the hybrid sample and $80 \AA$ for the calcined silica sample. For higher concentrations in surfactant, lamellar morphology dominates progressively leading to dense structures after calcination. GISAXS was performed on the as-synthesized film including $45 \mathrm{wt} \%$ copolymer to gain a more thorough description of the mesostructure. As shown in Figure $\mathbf{5}$, three almost homogeneous ellipses are obtained; the extremities of their major axis correspond to the peaks indexed as (100), (200) and (210) in the diffractogram. The observation of such ellipses instead of circles is expected in deposited films due to remaining in-plane constraints. In mesoporous materials, the combination of an important shrinkage due to the solvent evaporation and poor mechanical properties makes this effect especially pronounced. The film consists in 2D-hexagonal domains displaying a random orientation of the denser (100) plane (although the cylinders remain probably parallel to the substrate). This can be deduced from the homogeneity of the diffraction features. During the shrinkage phase, the component of any distance parallel to the substrate remains locked to its original value while the perpendicular one is free to contract. In these conditions no domain remains strictly speaking 2D-hexagonal but generally becomes oblique. Determined from the pattern in Figure 5, the parallel and perpendicular to the plane inter-cylinder distances are respectively $137 \AA$ and $96 \AA$. 
Solid-state NMR. The three silica/surfactant hybrid samples have been analyzed by ${ }^{29} \mathrm{Si}$ and ${ }^{1} \mathrm{H}$ solid state NMR spectroscopy. ${ }^{29}$ Si MAS-DEC spectra were obtained to determine a quantitative value of the condensation degree (CD) not accessible with a CP-MAS method. The spectra are depicted in Figure S3 and the relative intensities of the $\mathrm{Q}_{n}$ siloxane species are summarized in Table $\mathbf{S 1}$. The ${ }^{29} \mathrm{Si}$ spectra are dominated by $Q_{3}$ species and to a minor extent by $Q_{4}$ species whatever the $P 123$ concentration. In addition, all the as-synthesized samples exhibit a similar CD ranging from $75-79 \%$. This result clearly supports the effectiveness of the photoacid-catalyzed sol-gel step performed at a temperature very close to room temperature. In addition, it shows the limited role played by the surfactant content on the condensation reactions. Consequently, an additional stiffening of the network upon heating (usually prior to the calcination step) does not appear as necessary. By comparison, ${ }^{29} \mathrm{Si}$ NMR spectra of as-synthesized EISA films generally exhibit only a mixture of $\mathrm{Q}_{2}$ and $\mathrm{Q}_{3}$ species, corresponding to a $C D$ of approx. $50-60 \%$. Several weeks to several months of ageing are found to be necessary to form the first $Q_{4}$ species [36]. After thermal calcination, the $C D$ increases slightly to $85-87 \%$, suggesting a highly condensed network.

\subsection{Investigation of the photoinduced mesotructuration process}

Figure 6 shows the temporal evolution of the IR transmission spectra throughout irradiation as a 3D plot for the film containing $45 \mathrm{wt} \% \mathrm{P} 123$. Before UV exposure $(t=0 \mathrm{~s})$, the low signal of the $\mathrm{OH}$ stretching modes $\left(\approx 3400 \mathrm{~cm}^{-1}\right)$ only results from water retained by the amphiphilic block copolymer, thus establishing the absence of hydrolyzed products. Additionally, the distinctive and sharp symmetric $\mathrm{CH}_{3}$ stretching band at $2848 \mathrm{~cm}^{-1}\left(\mathrm{U}_{\text {sym }}\left(\mathrm{OCH}_{3}\right)\right)$ supports the presence of hydrolysable methoxysilyl groups $\left(\mathrm{SiOCH}_{3}\right)$. Four other bands are also visible in the $2800-3000 \mathrm{~cm}^{-1}$ range, which are assigned to $\mathrm{CH}$ stretching vibration from P123 and PDMOS. More precisely, the 2872, 2917, 2949 and $2970 \mathrm{~cm}^{-1}$ bands are attributed to $\mathrm{CH}_{2}$ symmetric, $\mathrm{CH}_{3}$ symmetric, $\mathrm{CH}_{2}$ antisymmetric and $\mathrm{CH}_{3}$ antisymmetric vibrations. Hydrolysis is first manifested by the formation of $\mathrm{Si}-\mathrm{OH}$ groups, which 
strongly absorb at $3700-3200 \mathrm{~cm}^{-1}$ due to the stretching of the $\mathrm{OH}-\mathrm{O}$ bonds, with the possible contribution of water by-products which may absorb as the film becomes more hydrophilic with the formation of silanol groups. Second, there is a gradual conversion of the methoxysilyl groups, resulting in the progressive disappearance of the $\mathrm{v}_{\text {sym }}\left(\mathrm{OCH}_{3}\right)$ band, which has completely vanished below the noise level after $12 \mathrm{~min}$ irradiation. After complete hydrolysis, further condensation reactions take place as indicated by the growth of the asymmetric stretching of Si-O-Si bonds arising as a broad massif in the range $1000-1260 \mathrm{~cm}^{-1}$ (not visible in Figure 6) [37].

Figure 7 relates hydrolysis degree obtained from the integration of $\mathrm{v}_{\text {sym }}\left(\mathrm{OCH}_{3}\right)$ band (left plot) to XRD patterns (right plot) for a series of samples containing 45 wt\% P123 and taken at regular irradiation times. As expected, the XRD pattern presents no signal at $t=0$, suggesting an initial isotropic medium. Therefore, PDMOS is a good enough solvent of P123 to prevent formation of mesophases. At $t=5 \mathrm{~min}$, when hydrolysis degree reaches $26 \%$, the first poorly meso-organized structures appear in the XRD pattern at 0.5 and $\sim 2.452^{\circ} \theta$. As a result, one can assume that amorphous-to-order transition is triggered by a sufficient concentration of silanol groups (or hydroxyl-containing compounds in general) within the film. Under these specific conditions, the higher polarity of the medium becomes conducive to surfactant self-assembly. Unfortunately, the peaks associated to these proto-mesophases cannot be properly indexed to match a defined phase. It is only when hydrolysis is almost complete ( $\mathrm{t}=10 \mathrm{~min}, 87 \%$ ), that the XRD pattern can be clearly indexed to a 2D hexagonal structure $\left(d_{100}=2 d_{200}=\sqrt{7} d_{210}\right)$. For longer exposure times, the XRD patterns exhibit somewhat a similar profile but the main peaks shifts gradually towards the high angles: $0.972^{\circ} \theta$ at $t=10 \mathrm{~min}$ and $1.112^{\circ} \theta$ at $\mathrm{t}=40 \mathrm{~min}$ for the (001) reflection. This suggests a contraction of the siloxane network due to further condensation reactions. In addition, the (200) and (210) reflections become less intense. We hypothesize that the internal constraints and deformations caused by the condensation reactions are likely to affect the mesostructure homogeneity. The corollary is an overall broadening of the XRD peaks, leading to poorly resolved (200) and (210) peaks in the final material. 
The combination of FTIR and XRD data is instrumental in establishing a light-induced self-assembly mechanism, which has been sketched in Scheme 1. Upon UV radiation, the PAG photolysis causes the generation of Brönsted acid of the structure $\mathrm{H}^{+} \mathrm{PF}_{6}{ }_{6}$. These protonic superacids are thought to play a prominent role in the catalysis of the sol-gel process. Hydrolysis of PDMOS induces a polarity change in the medium from a hydrophobic to a hydrophilic environment, thus fostering the formation of lyotropic mesophases in which segregated PEO blocks are in contact with silica, while the PPO blocks are preferentially located in the core of the mesophases. By contrast with results reported in the literature on photoinduced micellization [38], the driving force in the present process is not the change of copolymer structure to make it amphiphilic, but the modification of the solvation properties (the change from hydrophobic alkoxy precursor to hydrophilic silanol species).

\subsection{Influence of key parameters}

In order to optimize the synthesis conditions, the effect of $\mathrm{RH}$ and film thickness have been investigated.

Relative Humidity. The hygrometric UV chamber enables to vary the RH value from $30 \%$ to $90 \%$, and assess the effect of this very important parameter on hydrolysis rate and nanostructuration. Keeping the irradiation time unchanged ( $30 \mathrm{~min}$ ), all the samples prepared with $45 \mathrm{wt} \% \mathrm{P} 123$ showed complete hydrolysis (FTIR data) even under the lowest humidity conditions ( $\mathrm{RH}=30 \%$ ). Figure 8 is a series of XRD patterns for the as-synthesized copolymer/silica films obtained at different RH. At intermediate $\mathrm{RH}(50-70 \%)$, a 2D hexagonal mesophase dominates as was mentioned earlier. At the lowest $\mathrm{RH}$ values (30 - $40 \%$ ), a multiphasic system is rather formed. The low angle signals appearing in this case may reflect disordered worm-like mesostructures co-existing with some residual hexagonal structures. Accordingly, a decrease of $\mathrm{RH}$ may cause a reduction of the volume of the hydrophilic domain due to less water absorption inside the film. This may have a direct effect on the self-organization ability, and also on the mesostructure geometry. Higher RH (80 - 90\%) results in a 
single broad peak, showing a much lower intensity. This result may seem surprising because a high water concentration is supposed to be beneficial to mesotructuration and to the formation of highly curved mesophases $[39,40]$. However, under these conditions, the film presents cracks and even signs of delamination. Our hypothesis is that high humidity leads to film dewetting, thereby disturbing the self-assembly process and affecting the film mechanical integrity.

Film Thickness. The thickness of the PDMOS/P123 film containing 45 wt\% of templating agent has been progressively decreased from $3.6 \mu \mathrm{m}$ to $0.7 \mu \mathrm{m}$ by adjusting the acetone content (see composition in Table S2). Film thickness is a key parameter for self-assembly for two reasons. Firstly, thickness may affect the water vapor permeation rate. Here, water is both a reactant (hydrolysis) and a driver of mesostructuration (solvation), implying complex interactions between the permeant and the polymer film [32]. Secondly, a thinner film increases the surface-to-volume ratio of the film, resulting in a stronger influence of the substrate on mesophase orientation. As depicted in Figure 9 (left plot), the net effect of a film thickness reduction is particularly significant on the XRD patterns of the as-synthesized films. Thinner films lead to more intense and resolved peaks despite a lower analyzed sample volume. Our assumption is that a thinner film avoids water concentration gradient through the film leading to more homogeneous film in terms of composition and organization. Additionally, there is probably a stronger orientating and ordering effect of the substrate as the film thickness decreases as the proportion of mesostructure located in the bulk, i.e. far from the interfacial region, diminishes. The interpretation of the XRD data of the calcined film (Figure 9, right plot) is more difficult as the reflections are broader and less intense, but other techniques support a higher level of organization for the thinner film $(0.7 \mu \mathrm{m})$. TEM pictures clearly show a high proportion of highly ordered hexagonal structures in this sample (Figure S4). In addition, the GISAXS patterns of the as-synthesized and calcined films displayed in Figure $\mathbf{1 0}$ show that the diffraction ellipses display now strong reinforcements compared to the thicker sample (3.6 $\mu \mathrm{m}$, Figure 5$)$. This result can interpreted as a marked preferential orientation of the domains with the denser plane parallel to the 
substrate. As a final advantage, thinner calcined films also exhibit a better optical quality with fewer defects (see optical microscopy images in Figure S5).

\section{Conclusion}

An experimentally simple and eco-efficient photochemical set-up for synthesizing micrometer thick mesostructured copolymer/silica film on large surfaces has been described. Our photochemical set-up stands out by low energy consumption, inexpensive equipment and commercial starting materials. Exact control of radiation in terms of energy and time is ensured by a set of two UV fluorescent tubes. Sol-gel photopolymerization is performed inside a hygrometric chamber at a temperature close to ambient temperature. Investigation of the tricomponent mixture PDMOS (silica source), P123 (templating agent) and PAG has enabled to emphasize the role of P123 concentration on texture properties. At 55 wt\% P123, well condensed (> $75 \%$ ) 2D hexagonal silica/P123 mesostructures are formed with a preferential alignment of the hexagonally packed cylinders parallel to the substrate. The thermocalcined films showed a moderate specific surface area of $314 \mathrm{~m}^{2} \mathrm{~g}^{-1}$ because no micropores are actually detected. The best humidity conditions range between 40 and 50 $\%$, above this threshold film delamination occurs, below, a mixture of mesophases is generated. Decreasing film thickness below $1 \mu \mathrm{m}$ has found to be an efficient way to increase mesophase ordering and orientation, with also the benefit of forming crack-free films after the surfactant removal. To further increase the eco-efficiency credential of our photoprocess, the next step is to couple the present photoinduced mesostructuration with a photocalcination step in order to achieve a fully photochemical pathway to mesoporous films.

\section{Acknowledgements:}


This work was supported by the Fondation de l'Ecole Nationale Supérieure de Chimie de Mulhouse (France) which has granted a 3-year PhD scholarship to Mrs. Mathilde Sibeaud.

\section{References}

[1] C.T. Kresge, M.E. Leonowicz, W.J. Roth, J.C. Vartuli, J.S. Beck, Nature, 359 (1992) 710-712.

[2] J.S. Beck, J.C. Vartuli, W.J. Roth, M.E. Leonowicz, C.T. Kresge, K.D. Schmitt, C.T.W. Chu, D.H. Olson, E.W. Sheppard, J. Am. Chem. Soc., 114 (1992) 10834-10843.

[3] L. Malfatti, P. Falcaro, B. Marmiroli, H. Amenitsch, M. Piccinini, A. Falqui, P. Innocenzi, Nanoscale, 3 (2011) 3760-3766.

[4] B. Hatton, K. Landskron, W. Whitnall, D. Perovic, G.A. Ozin, Acc. Chem. Res., 38 (2005) 305-312.

[5] M.E. Davis, Nature, 417 (2002) 813-821.

[6] A. Stein, B.J. Melde, R.C. Schroden, Adv. Mater. (Weinheim, Ger.), 12 (2000) 1403-1419.

[7] J.W. Zhang, G.P. Zhang, Y.J. Gao, R. Sun, C.P. Wong, J. Mater. Sci., 51 (2016) 7966-7976.

[8] Y.F. Lu, R. Ganguli, C.A. Drewien, M.T. Anderson, C.J. Brinker, W.L. Gong, Y.X. Guo, H. Soyez, B. Dunn, M.H. Huang, J.I. Zink, Nature, 389 (1997) 364-368.

[9] C. Sanchez, P. Belleville, M. Popall, L. Nicole, Chem. Soc. Rev., 40 (2011) 696-753.

[10] N. Baccile, F. Babonneau, B. Thomas, T. Coradin, J. Mater. Chem., 19 (2009) 8537-8559.

[11] C. Gerardin, J. Reboul, M. Bonne, B. Lebeau, Chem. Soc. Rev., 42 (2013) 4217-4255.

[12] T. Kimura, Chem. Rec., 16 (2016) 445-457.

[13] R. Keshavarzi, V. Mirkhani, M. Moghadam, S. Tangestaninejad, I. Mohammadpoor-Baltork, Langmuir, 31 (2015) 11659-11670.

[14] P. Innocenzi, L. Malfatti, Chem. Soc. Rev., 42 (2013) 4198-4216.

[15] R. Schwalm, UV Coatings Basics, Recent Developments and New Applications, First ed., Elsevier, Amsterdam, The Netherlands, 2007.

[16] H.D. Paz-Simon, A. Chemtob, F. Crest, C. Croutxe-Barghorn, L. Michelin, L. Vidal, S. Rigolet, B. Lebeau, RSC Adv., 2 (2012) 11944-11952.

[17] H. De Paz-Simon, A. Chemtob, C. Croutxé-Barghorn, S. Rigolet, L. Michelin, L. Vidal, B. Lebeau, J. Phys. Chem. C, 118 (2014) 4959-4966.

[18] F.G. Liu, W.J. Yang, Y. Wang, X.X. Xue, H. Yang, Chem. Lett., 43 (2014) 720-722.

[19] M. Sibeaud, C. Croutxe-Barghorn, S. Rigolet, L. Michelin, L. Josien, L. Vidal, B. Lebeau, M. Worner, A. Chemtob, Rsc Adv., 6 (2016) 65047-65054.

[20] H. Nagasawa, M. Nishibayashi, M. Kanezashi, T. Yoshioka, T. Tsuru, Rsc Adv., 7 (2017) 7150-7157.

[21] W. Li, Y.G. Wu, S.K. Shen, S.F. Song, D.D. Hu, J. Phys. Chem. B, 120 (2016) 9513-9522.

[22] E. Maetz, C. Croutxé-Barghorn, C. Delaite, X. Allonas, Polym. Chem., 7 (2016) 7383-7390.

[23] L.Y. Song, Q. Ye, X.P. Ge, A. Misra, C. Tamerler, P. Spencer, Rsc Adv., 6 (2016) 52434-52447. 
[24] D. Massiot, F. Fayon, M. Capron, I. King, S. Le Calvé, B. Alonso, J.-O. Durand, B. Bujoli, Z. Gan, G. Hoatson, Magn. Reson. Chem., 40 (2002) 70-76.

[25] C. Cowan-Ellsberry, S. Belanger, P. Dorn, S. Dyer, D. McAvoy, H. Sanderson, D. Versteeg, D. Ferrer, K. Stanton, Crit. Rev. Environ. Sci. Technol., 44 (2014) 1893-1993.

[26] E.K. Richman, T. Brezesinski, S.H. Tolbert, Nat. Mater., 7 (2008) 712-717.

[27] M. Thommes, K.A. Cychosz, Adsorption, 20 (2014) 233-250.

[28] R. Ryoo, C.H. Ko, M. Kruk, V. Antochshuk, M. Jaroniec, J. Phys. Chem. B, 104 (2000) 11465-11471.

[29] A. Galarneau, H. Cambon, F. Di Renzo, F. Fajula, Langmuir, 17 (2001) 8328-8335.

[30] A. Galarneau, H. Cambon, F. Di Renzo, R. Ryoo, M. Choi, F. Fajula, New J. Chem., 27 (2003) 73-79.

[31] H. De Paz, A. Chemtob, C. Croutxé-Barghorn, S. Rigolet, B. Lebeau, Microporous Mesoporous Mater., 151 (2012) 88-92.

[32] Y. Lu, R. Ganguli, C.A. Drewien, M.T. Anderson, C.J. Brinker, W. Gong, Y. Guo, H. Soyez, B. Dunn, M.H. Huang, J.I. Zink, Nature, 389 (1997) 364-368.

[33] P.C.A. Alberius, K.L. Frindell, R.C. Hayward, E.J. Kramer, G.D. Stucky, B.F. Chmelka, Chem. Mater., 14 (2002) 3284-3294.

[34] H. Miyata, Y. Kawashima, M. Itoh, M. Watanabe, Chem. Mater., 17 (2005) 5323-5327.

[35] D. Zhao, Y. Wan, W. Zhou, Structural Characterization Methods, Ordered Mesoporous Materials, WileyVCH Verlag GmbH, pp. 117-151.

[36] D. Grosso, F. Cagnol, G.J.d.A.A. Soler-Illia, E.L. Crepaldi, H. Amenitsch, A. Brunet-Bruneau, A. Bourgeois, C. Sanchez, Adv. Funct. Mater., 14 (2004) 309-322.

[37] H. De Paz, A. Chemtob, C. Croutxé-Barghorn, D. Le Nouen, S. Rigolet, J. Phys. Chem. B., 116 (2012) 52605268

[38] J.-F. Gohy, Y. Zhao, Chem. Soc. Rev., 42 (2013) 7117-7129.

[39] G. Soler-IIlia, E.L. Crepaldi, D. Grosso, D. Durand, C. Sanchez, Chem. Commun. (Cambridge, U. K.), (2002) 2298-2299.

[40] V.N. Urade, L. Bollmann, J.D. Kowalski, M.P. Tate, H.W. Hillhouse, Langmuir, 23 (2007) 4268-4278. 
Table 1: Specific surface area, pore volumes and pore widths from the $\mathrm{N}_{2}$ adsorption experiments and TEM analysis

\begin{tabular}{|c|c|c|c|}
\hline $\begin{array}{l}\text { PDMOS/P123 } \\
\text { wt. ratio }\end{array}$ & $1 / 0.25$ & $1 / 0.45$ & $1 / 0.55$ \\
\hline $\begin{array}{c}\text { BET specific surface } \\
\text { area }^{a}\left(\mathrm{~m}^{2} \mathrm{~g}^{-1}\right)\end{array}$ & 119 & 191 & 314 \\
\hline $\begin{array}{l}\text { Total volume of pores } \\
\qquad\left(\mathrm{cm}^{3} \mathrm{~g}^{-1}\right)\end{array}$ & 0.14 & 0.30 & 0.37 \\
\hline $\begin{array}{l}\text { t-Plot micropore } \\
\text { volume }^{\mathrm{a}}\left(\mathrm{cm}^{3} \mathrm{~g}^{-1}\right)\end{array}$ & - & - & 0.03 \\
\hline $\begin{array}{l}\text { BJH desorption pore } \\
\text { width }(\AA ̊ \cap)\end{array}$ & 39 & $38 / 43$ & $43 / 48$ \\
\hline $\begin{array}{c}\text { Pore width }^{\text {b }} \text { (from TEM } \\
\text { data) ( }(\AA)\end{array}$ & 34 & 41 & 50 \\
\hline
\end{tabular}

${ }^{a}$ determined by $\mathrm{N}_{2}$ adsorption measurements, ${ }^{\mathrm{b}}$ determined by TEM data 

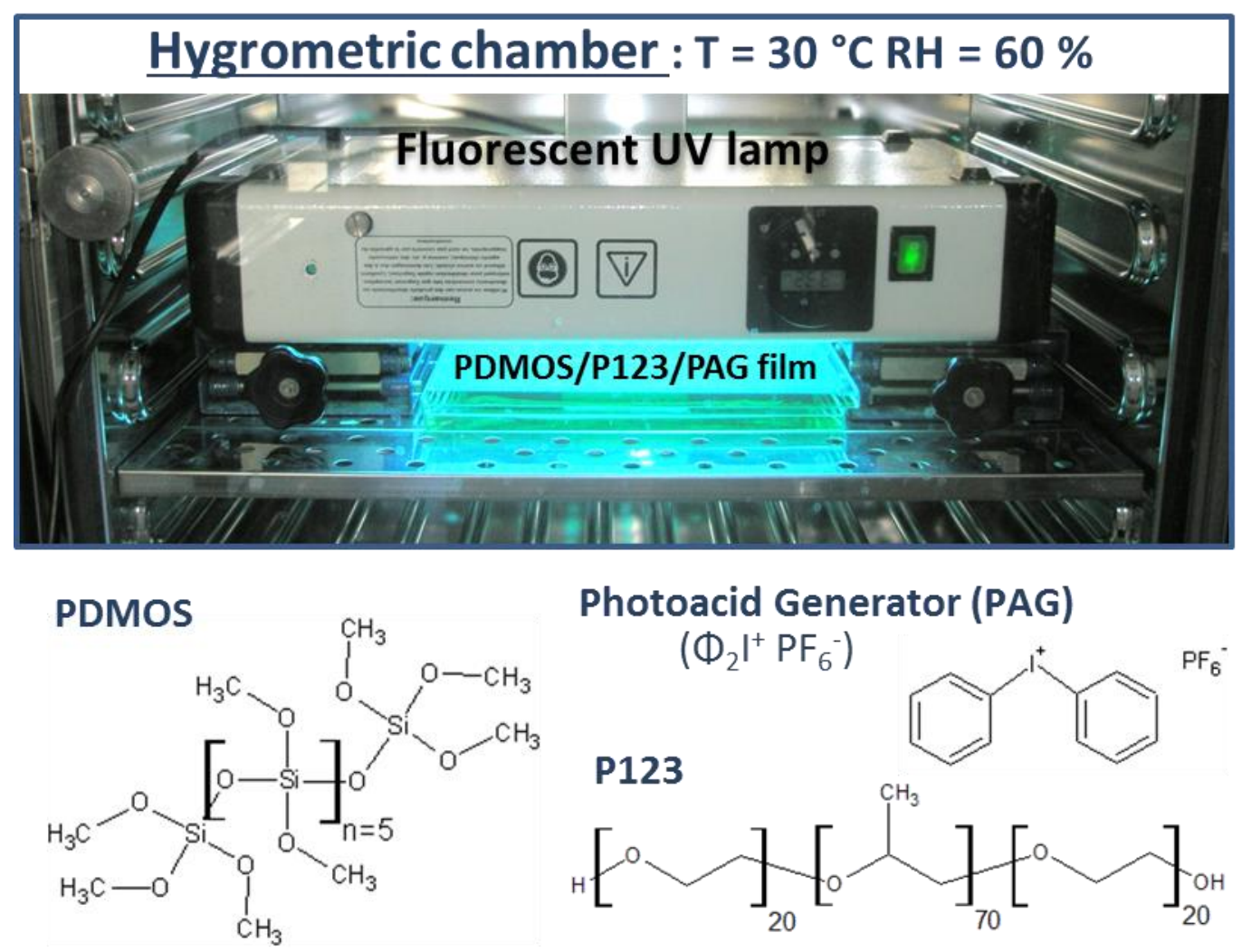

\section{Photoacid Generator (PAG)}

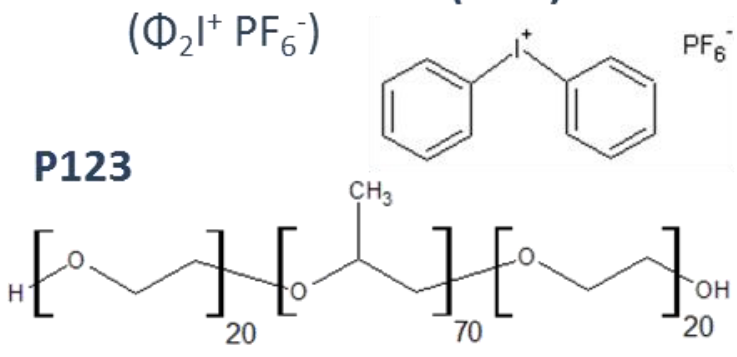

Figure 1: UV irradiation set up (at the top) and reactants (at the bottom). Irradiation is provided by a compact system composed of two fluorescent UV tubes whose radiation spans from 280 to $380 \mathrm{~nm}$. A total irradiance of approx. $3 \mathrm{~mW} \mathrm{~cm}^{-2}$ reaches the liquid PDMOS/P123/PAG film placed beneath at a distance of $6 \mathrm{~cm}$. 


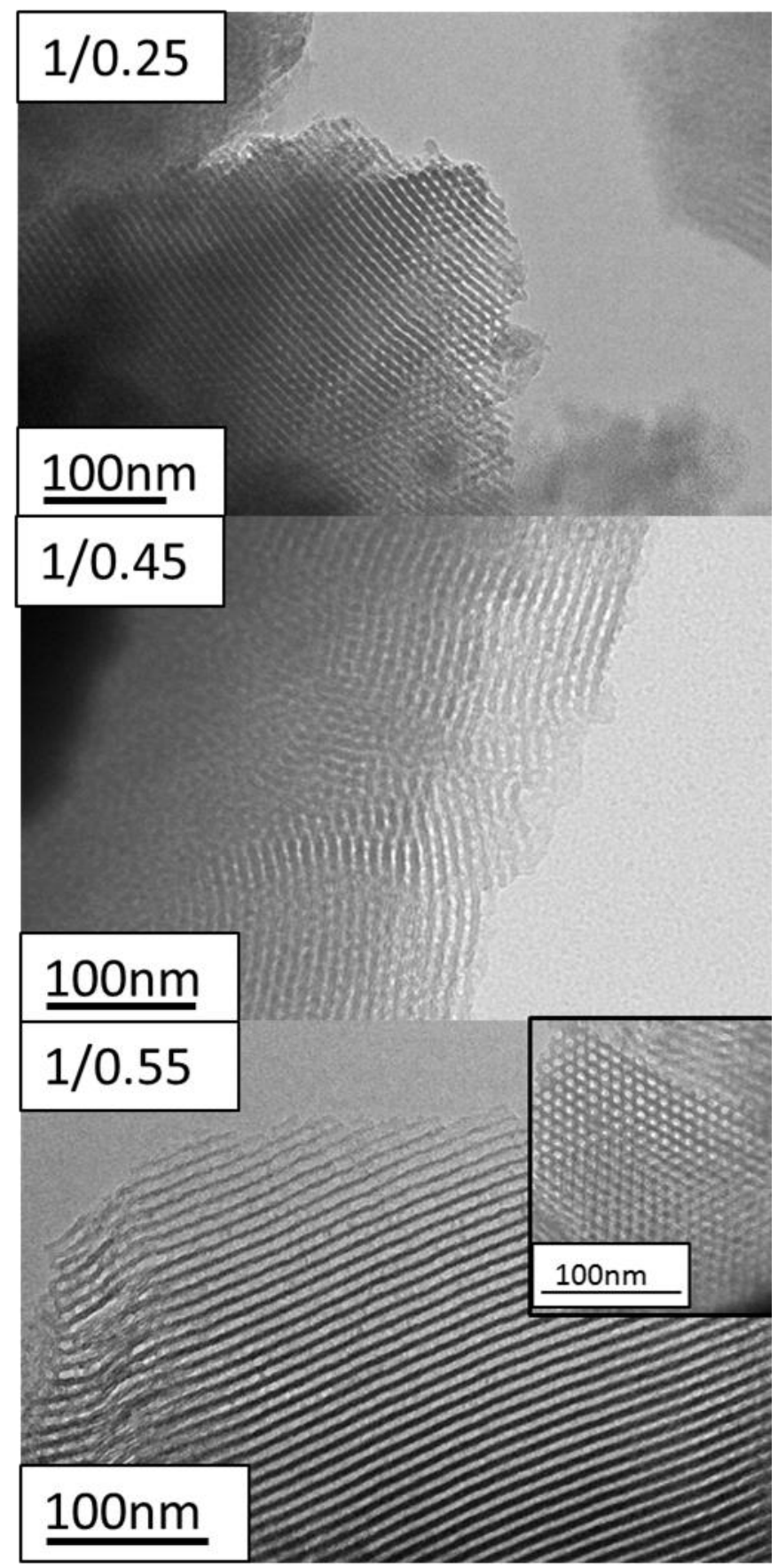

Figure 2: TEM images of calcined silica films prepared at three different P123/PDMOS weight ratios indicated in the upper left-hand corner of each image. 


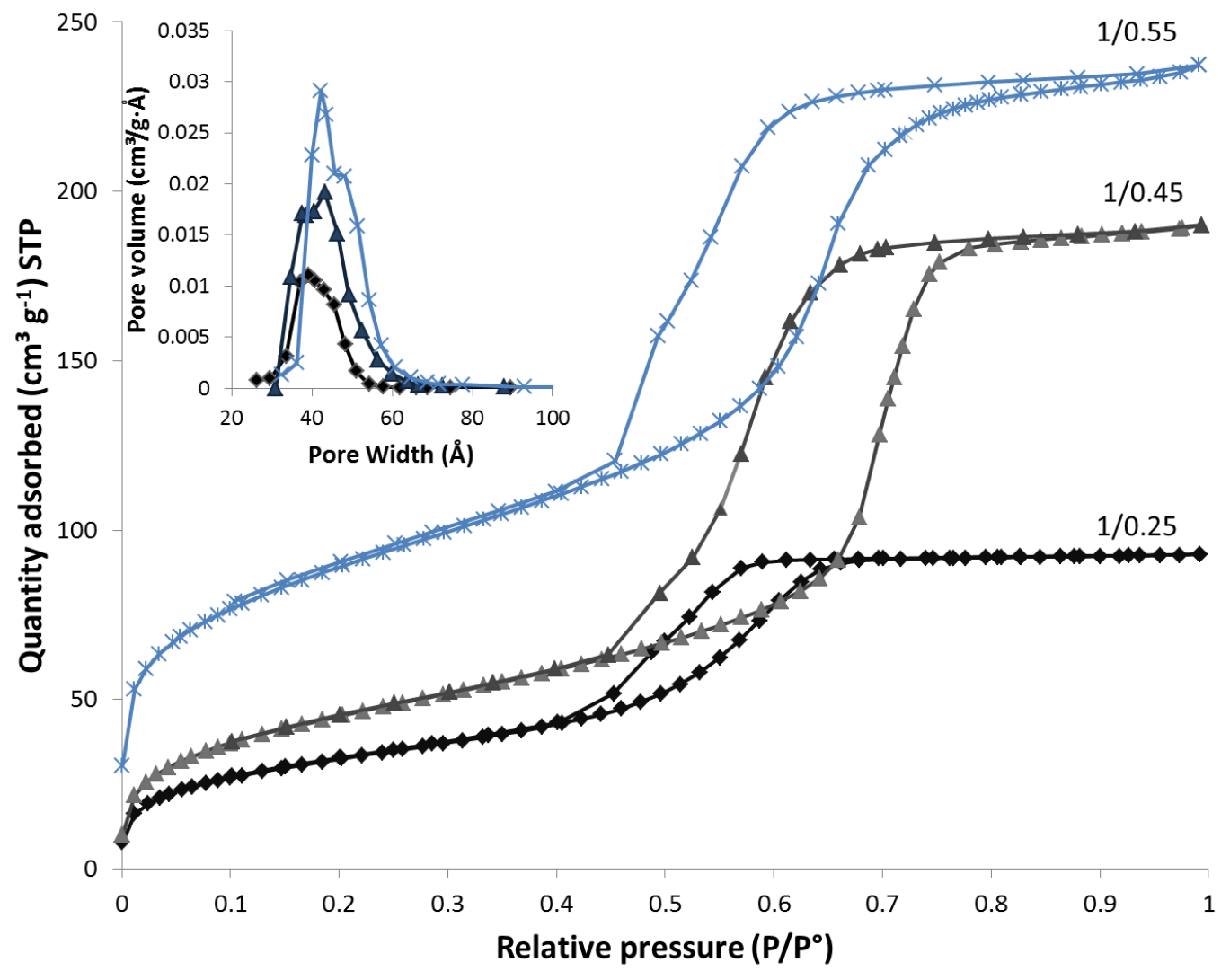

Figure 3: $\mathrm{N}_{2}$ adsorption isotherms and $\mathrm{BJH}$ pore size distribution (insert) determined from desorption branches for silica samples prepared at three different PDMOS/P123 weight ratios: 1/0.25, 1/0.45 and $1 / 0.55$.

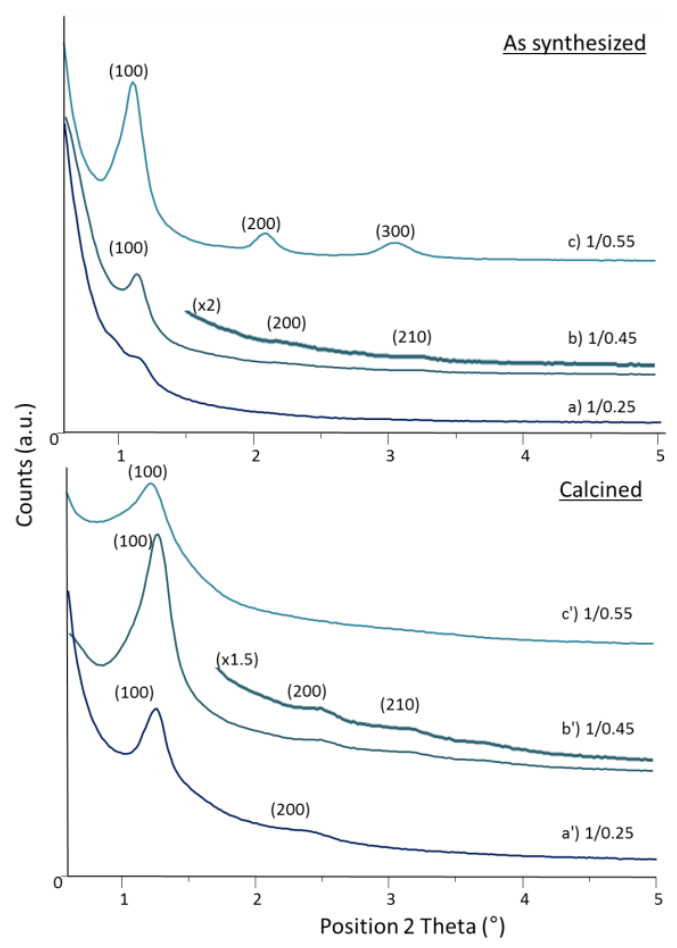

Figure 4: XRD patterns of as-synthesized silica/surfactant films and calcined silica films prepared at different PDMOS/P123 weight ratios. 


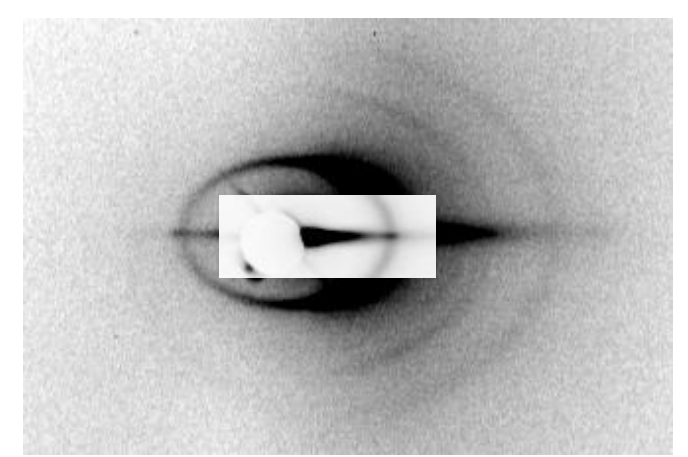

Figure 5: GISAXS pattern of as-synthesized P123/silica film ( $x=0.45$, film thickness: $3.6 \mu \mathrm{m}$ )

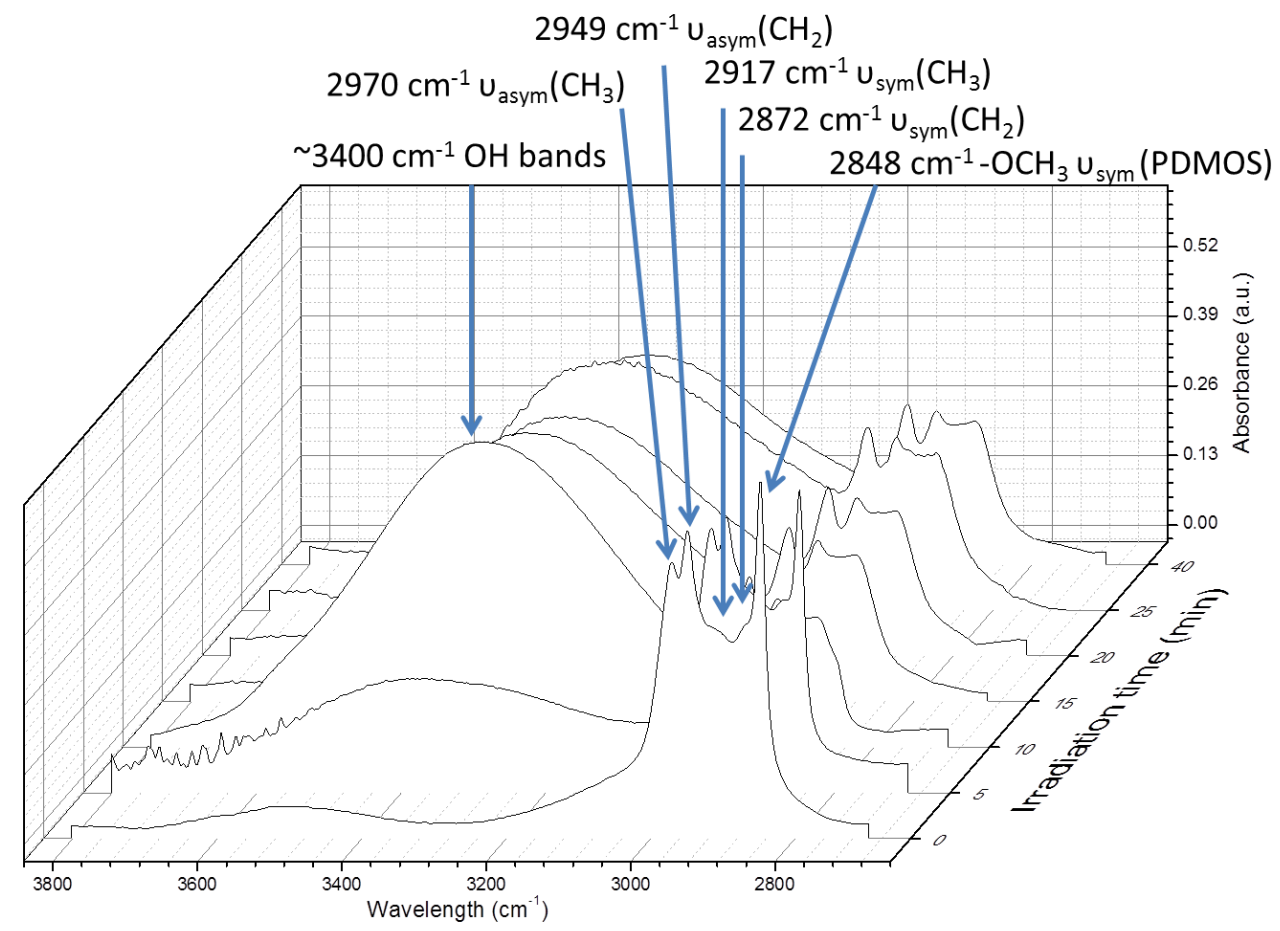

Figure 6: FTIR spectra as a function of the irradiation time for PDMOS/P123=1/0.45. 


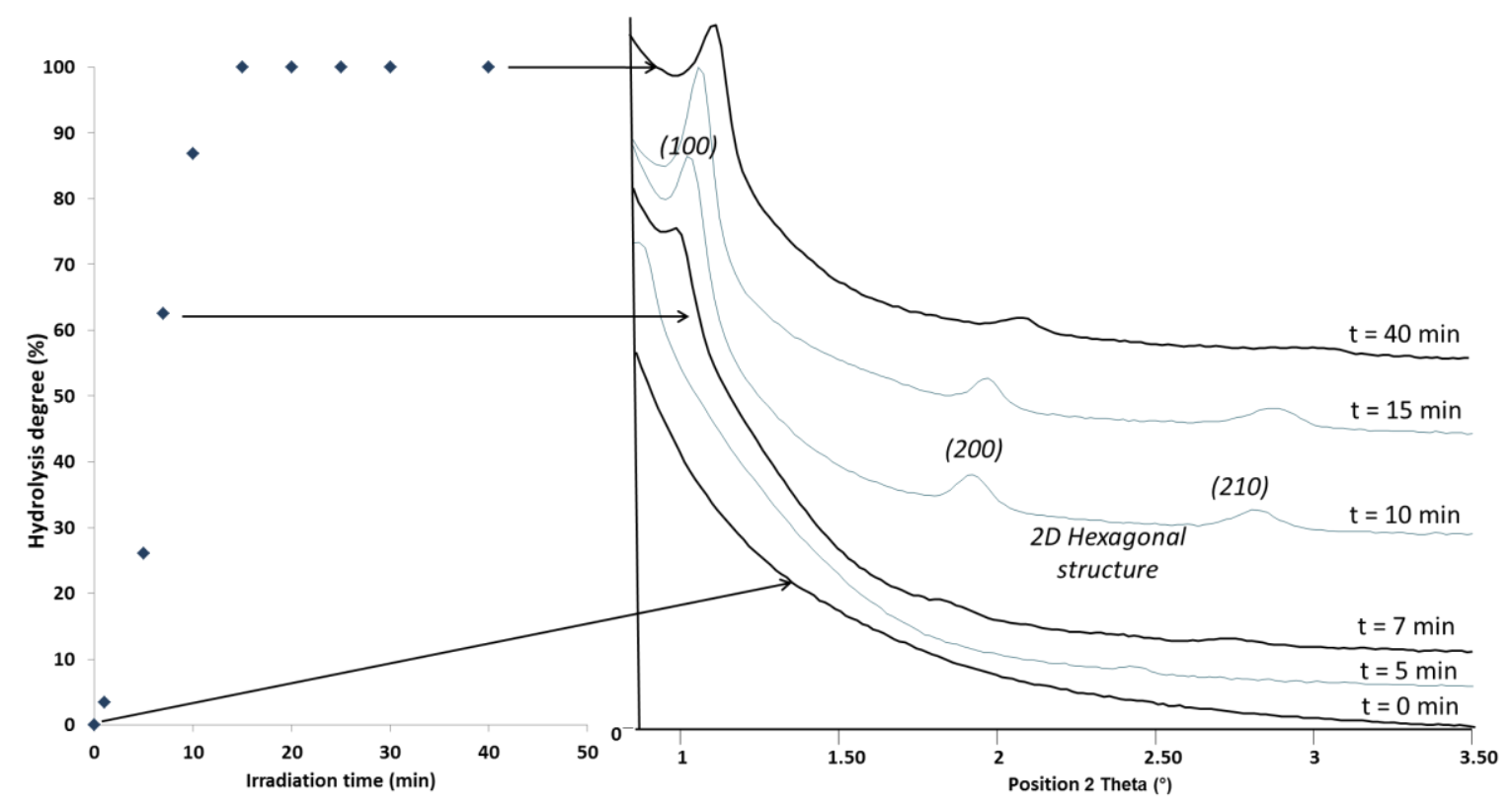

Figure 7: Hydrolysis rate as a function of the irradiation time (left) and the corresponding XRD patterns (right). PDMOS/P123=0.45.

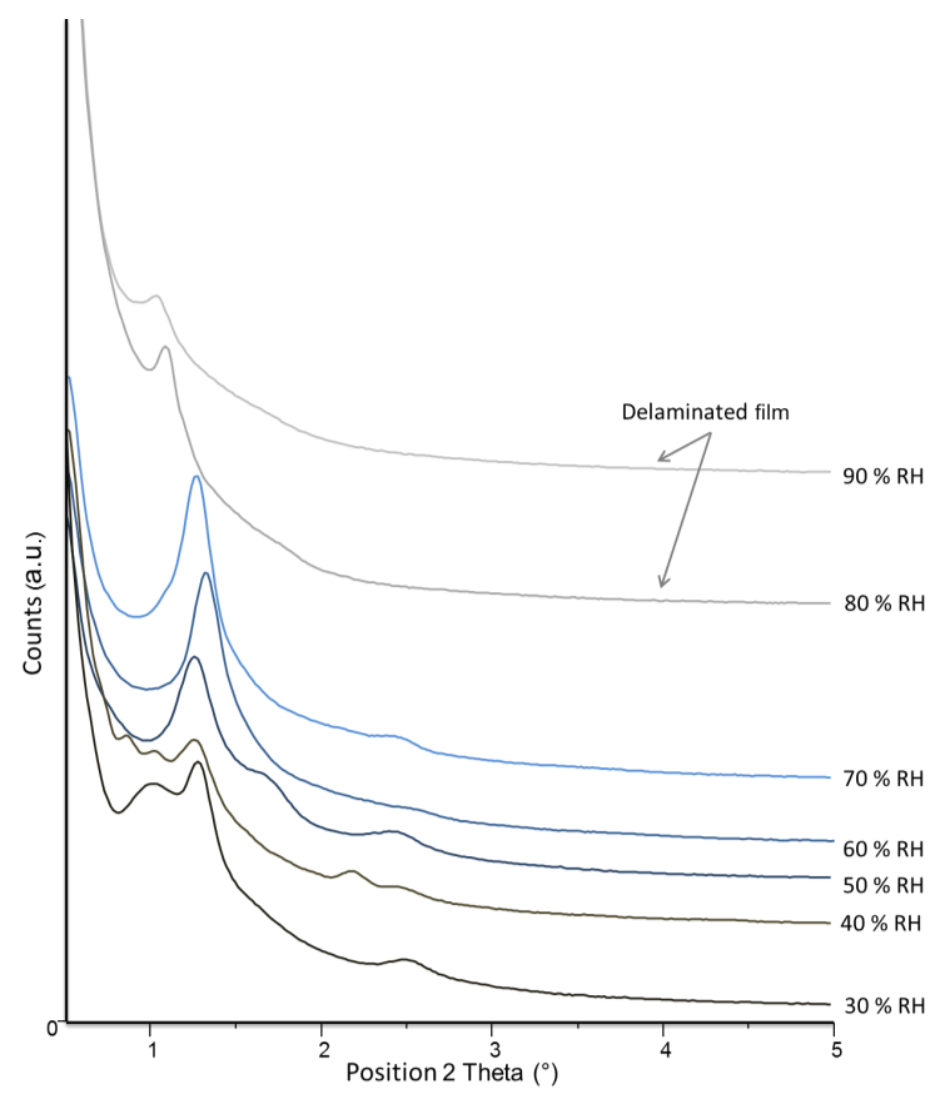

Figure 8: XRD pattern of PDMOS/P123 (1/0.45) calcined films as a function of RH. 


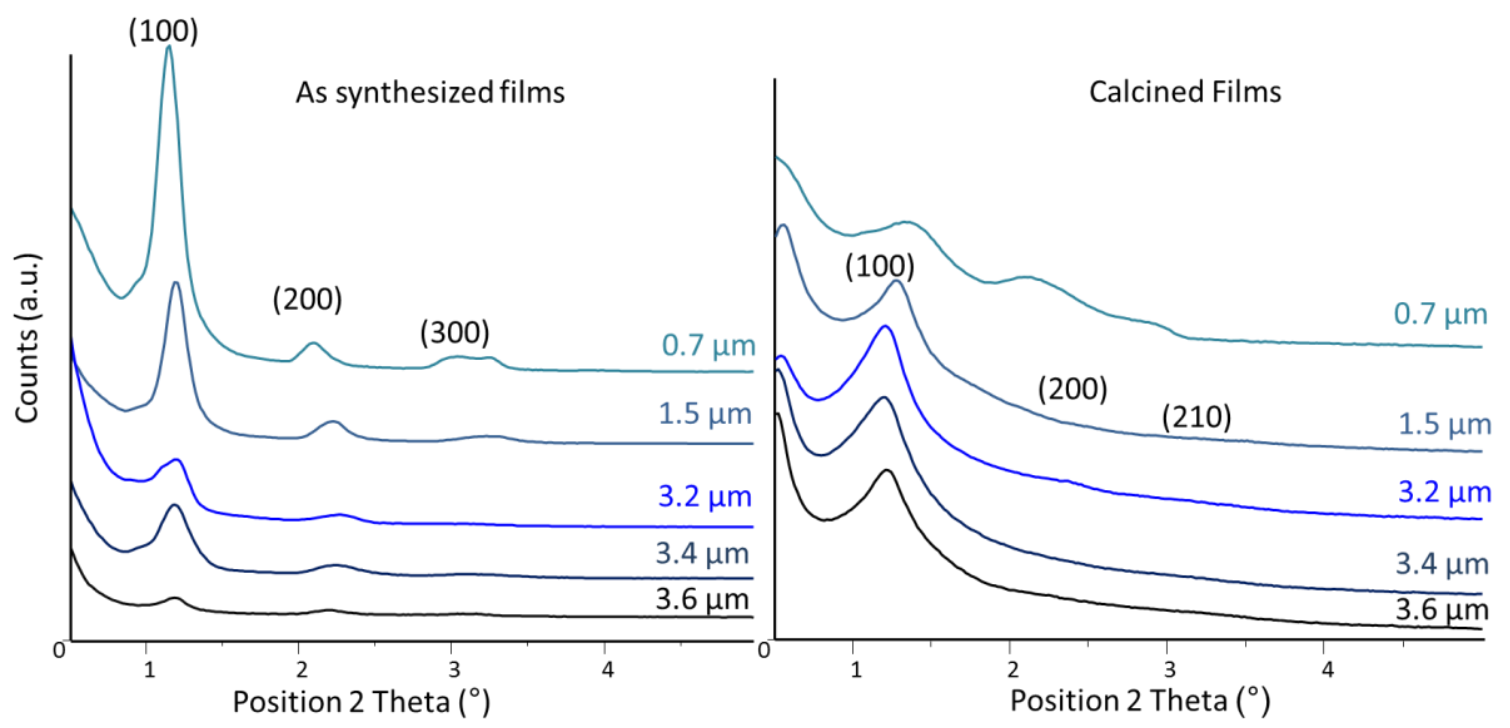

Figure 9: Effect of film thickness on XRD patterns of as-synthesized (left) and calcined (right) silica prepared with $45 \mathrm{wt} \% \mathrm{P} 123(\mathrm{x}=0.45)$.

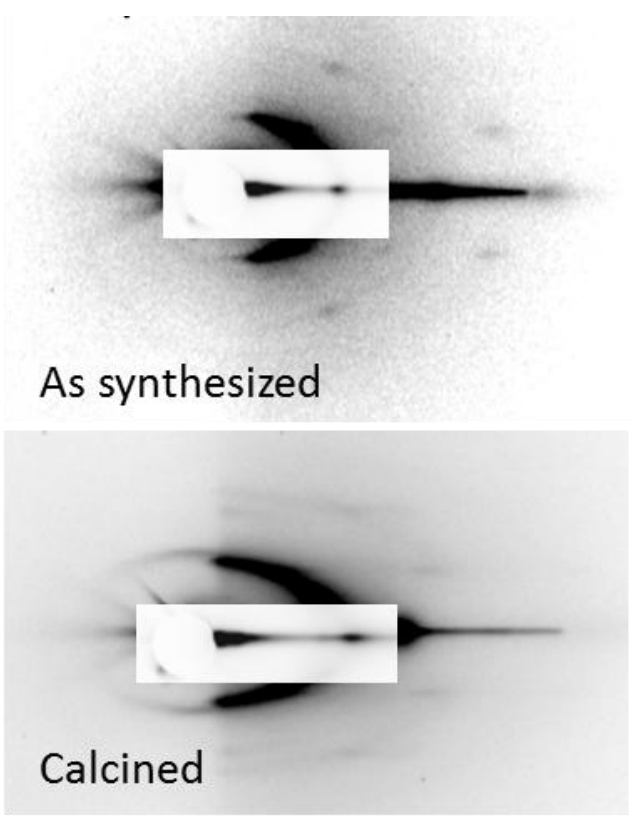

Figure 10: GISAXS patterns of as synthesized and calcined silica films prepared with $45 \mathrm{wt} \% \mathrm{P} 123$ copolymer (film thickness: $0.7 \mu \mathrm{m}$ ). Due to the asymmetric contraction of the film resulting from solvent evaporation, the oriented domains are strictly speaking centered rectangular and not 2Dhexagonal. 
: PDMOS<smiles>CO[Si](C)(OC)O[Si](C)(OC)OC</smiles>

$\star$ : PAG $\left(\Phi_{2} 1^{+} P F_{6}^{-}\right)$<smiles>[C](c1ccccc1)c1ccccc1</smiles>

$\mathrm{PF}_{6}^{-}$

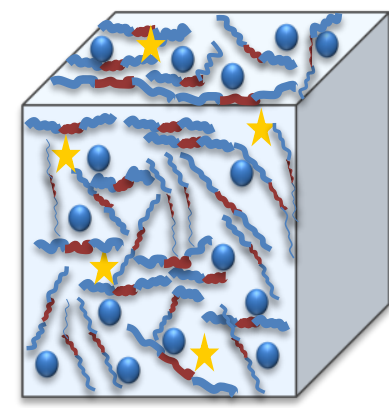

Isotropic Formulation

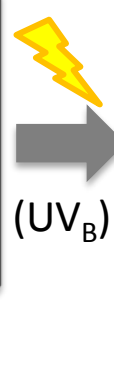

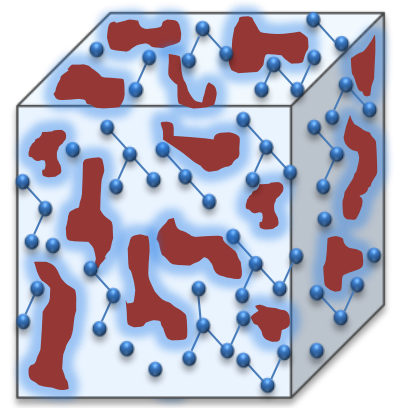

Phase separation

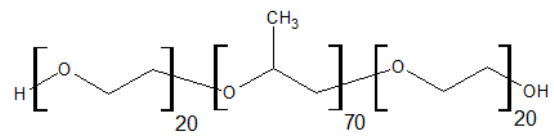

: Pluronic 123

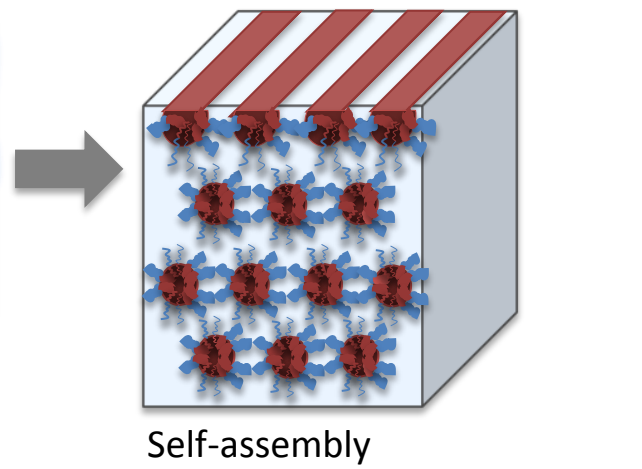

Scheme 1. Light controlled self-assembly mechanism 